\title{
Electronic structure computations of Newton Black Films
}

\author{
Fernando Bresme, ${ }^{* a}$ and Emilio Artacho ${ }^{* b}$ \\ Received Xth $X X X X X X X X X X 20 X X$, Accepted $X$ th $X X X X X X X X X 20 X X$ \\ First published on the web Xth $X X X X X X X X X X 200 X$ \\ DOI: 10.1039/b000000x
}

We investigate using quantum density functional theory (DFT) the electronic structure of sodium dodecyl sulfate Newton Black Films (NBF), consisting of water sandwiched between surfactants surrounded by vacuum. We generate a classical trajectory of the film using full-atom empirical potentials. DFT theory is employed to investigate the electron density and electrostatic properties of the film. The electrostatic potential derived directly from the electron and nuclear densities shows an important drop towards the vacuum side, similar to recent findings for the water-vacuum interface. We discuss the physical significance of this potential drop. We also consider as an alternative definition of the electrostatic potential, the deformation charge potential (DCP), to quantify the electrostatic properties of the interfaces. The analysis of the DCP potential indicates there is charge separation at the surfactant alkyl tails, showing that the surfactant-vacuum surface has an excess of positive charge. This result is consistent with molecular dynamics simulations employing classical force-fields, which incorporate partial charges to model the surfactant alkyl chain. The DCP potential also gives evidence for the existence of electronic polarization in the hydrophobic region of the NBF, whose origin is connected to charge transfer between the surfactant tails and the head group. We suggest that the deformation charge potential provides a route to compare the electrostatic potentials obtained from DFT and emprirical computations. This idea is illustrated by computing the electrostatic potential of the water-vapor interface. We discuss the relevance of these results in the context of DFT computations of soft interfaces.

\section{Introduction}

Surfactants can self-assemble into a wide variety of complex structures ${ }^{1}$. At high concentrations they can form Black Films $(\mathrm{BF})$, consisting of an aqueous core stabilized by the surfactants. The stability of the BF is important in determining the stability of foams (gas-liquid dispersions) and emulsions (liquid-liquid dispersions) ${ }^{2}$, which are of practical interest in many industrial problems, from food processing to oil recovery.

It is now well established that BF can adopt two main structures. One of them, the Common Black Film (CBF), has a thickness that can vary from about $6 \mathrm{~nm}$ to $100 \mathrm{~nm}$. The second type of BF was already discussed by Hooke and Newton, who reported the formation of very thin regions in soap bubbles. This represents one of the first observations of the existence of Newton Black Films (NBF), whose thickness, of the order of $4 \mathrm{~nm}$, is much smaller than that of the CBF. The CBF to NBF transition is considered to be first order, and can be induced by adding salt to a CBF. Several investigations have exposed the different physical behavior of $\mathrm{CBF}$ and NBF films. The thickness of a CBF is sensitive to the

\footnotetext{
${ }^{a}$ Department of Chemistry, Imperial College London, London, SW7 2AZ, UK. Tel: +44 (0)207 594 5886; E-mail:fbresme@imperial.ac.uk

${ }^{b}$ Department of Earth Sciences, University of Cambridge, Downing Street, Cambridge CB2 3EQ, UK.Tel. +44 (0)1223 333480, Cambridge, UK; Email:ea245@cam.ac.uk
}

ionic strength ${ }^{3-5}$, and its dependence with salt concentration follows the 'double-layer' picture described by the PoissonBoltzmann (PB) theory ${ }^{1,6}$. Interestingly, the variation of the thickness of the NBF with salt concentration shows significant deviations from the double layer picture ${ }^{7}$, and in some cases it has been reported that the thickness is independent on the amount of added salt ${ }^{8}$. These observations indicate that the electrostatic interactions represented by the double layer theories, e.g., PB, do not operate in NBF. The discrete nature of the ionic charges and the finite size of the ions may become relevant in determining the properties of the film ${ }^{9}$. In addition, the water response, which is expected to deviate significantly from the dielectric continuum approximation adopted in the PB theory, should play a significant role in determining the electrostatic potential inside the film. Several theoretical studies have attempted to correct this deficiency of the PB theory by considering a non local dielectric response of confined water. We have recently shown, using classical computer simulations, that water exhibits an anomalous dielectric behavior in ionic Newton Black Films ${ }^{10}$. Under the confinement conditions found in NBFs, water molecules are highly polarized, in the sense that they adopt a preferred orientation. This polarization is not consistent with a macroscopic local relation between the polarization and the electrostatic field. This explains why the double layer approximation fails in NBFs, since it neglects the polarization contribution of the solvent.

The stability of NBFs cannot be explained with conven- 
tional van der Waals and electrostatic forces. New types of interactions have been proposed, namely, hydration forces ${ }^{11}$ which have been interpreted in terms of water polarization or reorientation in the confined region ${ }^{12,13}$ and steric forces ${ }^{14-16}$, arising from the thermal fluctuations of the surfaces. Both ideas lead to interactions that agree in magnitude and decay length with experiments, although the physical origin of these interactions is completely different. We have investigated this issue in ionic Newton Black Films. We found that the electrostatic fields arising from the anomalous dielectric behavior of confined water ${ }^{10}$ are quite different from the predictions of electrostatic theories of hydration forces ${ }^{12,17}$. This anomalous dielectric response of confined water may contribute to the repulsive forces ${ }^{18}$. This work considered classical models, and therefore neglected the possibility of electronic polarization.

In this article we report the first computational investigation of the electronic structure of a NBF. We note that the aim of our paper is not the optimization of empirical potentials using DFT computations. We deal with a more fundamental question, namely, for trajectories obtained from classical simulations, which account well for the thermal fluctuations, we want to investigate the effect of having realistic electron polarization and polarizability on the electrostatic properties of the black films. We have chosen as ionic surfactant, sodium dodecyl sulfate (SDS). SDS is a surfactant widely used in technological and industrial applications, and it can stabilize Newton Black Films in both foams ${ }^{8,19}$ and emulsions ${ }^{20}$. The comparison of X-Ray reflectivity ${ }^{8}$ and small angle neutron scattering experiments ${ }^{20}$ indicates that the thickness of the NBF formed in foams and emulsions is very similar. This suggests that the surfactant interactions mediated by the aqueous core are similar in both cases too. This is interesting because the NBF separates two phases with very different properties, air (foams) vs. oil (emulsions).

Several works have reported classical computer simulation investigations of SDS black film foams ${ }^{10,18,21-25}$. Simulation studies indicate that the equilibrium film contains between two and four water molecules per surfactant, and between two and three of these molecules are part of a solvation layer. This reinforces the idea that in the equilibrium structure of the film there is no bulk water, and the water properties should be dominated by the interfaces present in the film. Quantifying the electronic structure of aqueous solutions, and in particular water, under these confinement conditions is a problem of considerable relevance in Material and Earth sciences, e.g., watermineral interfaces, as well as atmospheric science ${ }^{26-28}$.

Early ab initio molecular dynamics simulations ${ }^{29-32}$ investigated the electronic structure of bulk water. Subsequent studies have shown that increasing the simulation time leads to an over-structuring of the liquid, with a concomitant drop in the diffusion coefficient of water ${ }^{33-36}$. More recently, the properties of the water surface have been analyzed using $a b$ ini- tio molecular dynamics 37,38 . It stems from these works that the surface potential of water is of the order of $+3 \mathrm{~V}$, much larger than the potential expected from experimental studies $\approx+0.1 \mathrm{~V}$, whereas the prediction of computer simulations using empirical force-fields is $\approx-0.5 \mathrm{~V}$. The origin of these discrepancies remains unclear, although it is likely that these three potentials correspond to different situations and therefore cannot be compared with each other.

The ab initio molecular dynamics simulations performed so far have considered about $10^{2}$ water molecules and simulation times between $10-10^{2}$ ps. The investigation of Newton Black Films introduces a number of challenges. A minimum system size, typically of the order of $10^{3}$ atoms, is needed to properly model the film structure. That system size is much larger than the ones used in routine DFT computations of materials. Similarly, long simulation times, typically in the nanosecond time-scale, are needed to sample the film structure. This makes almost impossible to perform an ab initio simulation with current state of the art computational facilities. In this work we overcome this problem by combining classical and DFT computations. Hence, we perform the sampling of the nuclear coordinates using classical molecular dynamics, and perform single point computations of selected configurations using DFT theory. In this way we quantify the electronic structure of the film and analyze polarization effects in the film structure.

\section{Methodology}

\subsection{Classical simulations}

The black film consisted of an aqueous core coated with sodium dodecyl sulfate surfactants and their corresponding counterions (sodium) (see Figure 1). We did not consider added salt in the simulations. The film was surrounded by a big vacuum gap in the direction normal to the film, $z$. The box length in that direction was $L_{z}=5 L_{x}=5 L_{y}$, where $L_{x}=L_{y}$ is the length of the box on the film plane.This prismatic box minimizes the interactions between the film and its replicas in the $z$ direction, making possible the simulation of the two dimensional film. The interface area, $L_{x} \times L_{y}$ was set to reproduce the surface area per surfactant, $33 \AA^{2}$, reported in XRay reflectivity experiments ${ }^{8}$. The DFT computations were performed using 32 surfactants, 16 per monolayer. Such a small system size was necessary to reduce the computational cost associated with the $a b$ initio calculations. This system size ensures that the cut-off is long enough to ensure adequate computations of the van der Waals component in the classical simulations, and also minimizes the periodic error associated to the use of periodic boundary conditions (see ref. ${ }^{39}$ ). We checked that the structure obtained from the simulations of this small 'patch' was consistent with that of a larger sys- 
tem, consisting of 128 surfactants, 64 per monolayer. For the smaller film we considered 136 water molecules, giving 4.25 water molecules per surfactant ${ }^{8,22}$. The total number of atoms for the small film was 1784 . We considered the same amount of water per surfactant for the simulations with 128 surfactants. All the simulations were performed at constant temperature, $300 \mathrm{~K}$, in the canonical ensemble, using a Nosé-Hoover thermostat with a coupling constant of $0.5 \mathrm{ps}$. The trajectory was integrated using the leap frog algorithm with a time step of $0.002 \mathrm{ps}$.

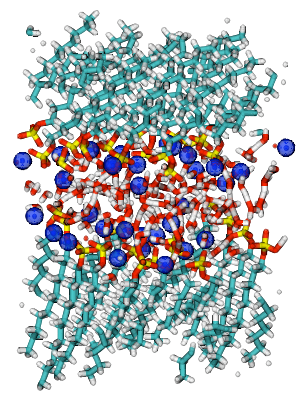

Fig. 1 Snapshot of the Sodium Dodecyl Sulfate black film investigated in this work. The large spheres represent the $\mathrm{Na}^{+}$ counterions, and water (middle of the film) and the surfactant molecules (above and below) are represented with sticks.

Water was modeled using the three site SPC/E model ${ }^{40}$. The $\mathrm{Na}^{+}$counterions were modeled as point particles using the Lennard-Jones (12-6) potential and one charge, $+1 e$ (see table 1). We considered a full atom model for the SDS surfactants. The model includes bonding, bending and torsional terms, as well as partial charges on the head group atoms. The partial charges in the head group were fitted to results obtained from previous quantum mechanics computations ${ }^{41}$. For the atoms in the alkane chains we considered two approaches, namely, zero charge (NQ-SDS) and partial charges (Q-SDS) as defined by the OPLS-AA force-field ${ }^{42}$. Although it has been shown that the partial charges have very little effect in the structure, thermodynamic and coexistence properties of alkanes ${ }^{43,44}$, they may add a contribution to the electrostatic potential of the surfactants in the NBF. Hence, introducing the partial charges provides a more direct check between the classical and $a b$ initio computations. The Lennard-Jones interactions were cutoff at $11.4 \AA$, and the Coulombic interactions were computed with the Ewald summation method using pa- rameters that ensured a typical relative error in the Coulombic energy of $10^{-6}$.

Table 1 Lennard Jones (12-6) parameters and partial charges employed in this work. The atoms in the aliphatic chain, $\mathrm{CH}_{2}$ and $\mathrm{CH}_{3}$ groups, were modeled as neutral atoms or with partial charges (numbers in brackets). See text for details.

\begin{tabular}{llll}
\hline Group & $\varepsilon(\mathrm{kJ} / \mathrm{mol})$ & $\sigma(\AA)$ & $\mathrm{q} / \mathrm{e}$ \\
\hline $\mathrm{Na}^{+}$ & 0.4179 & 2.5840 & 1.0 \\
$\mathrm{O}$ (water) & 0.6502 & 3.1660 & -0.8476 \\
$\mathrm{H}($ water $)$ & 0 & 0 & 0.4238 \\
$\mathrm{C}\left(\mathrm{CH}_{3}\right)$ & 0.4577 & 3.8160 & $0(-0.18)$ \\
$\mathrm{C}\left(\mathrm{CH}_{2}\right)$ & 0.4577 & 3.8160 & $0(-0.12)$ \\
$\mathrm{H}$ (alkane chain) & 0.0657 & 2.9740 & $0(0.06)$ \\
$\mathrm{C}$ (bonded to O-ester) & 0.4577 & 3.8160 & 0.137 \\
$\mathrm{O}$ (ester) & 0.8786 & 3.3224 & -0.4590 \\
$\mathrm{O}\left(\mathrm{SO}_{4}\right.$ group) & 0.8786 & 3.3224 & -0.654 \\
$\mathrm{~S}$ & 1.046 & 4.0 & 1.284 \\
\hline
\end{tabular}

The intramolecular interactions of the SDS surfactants were modeled using a combination of rigid bonds and a bending, $U_{\text {bend }}=k_{i j k} / 2\left(\theta_{i j k}-\theta_{e}\right)^{2}$ potential $^{45}$. The torsional potentials of the hydrocarbon chain, namely, C-C-C-C, C-C-C-H, and H$\mathrm{C}-\mathrm{C}-\mathrm{H}$, were modeled using the OPLS-AA forcefield ${ }^{42}$. For the dihedral angles involving the head group we considered the functional form, $U_{t o r}=A_{i j k l}\left[1+\cos \left(m \phi_{i j k l}+\delta\right)\right]\left(\mathrm{see}^{23}\right)$, with the parameters listed in table 2 .

Table 2 Intramolecular interactions used to model the SDS surfactants. Distances are given in $\AA$, angles in degrees and energy terms in $\mathrm{kJ} / \mathrm{mol}$.

\begin{tabular}{ll}
\hline Interacting Groups & Interaction parameters \\
\hline $\mathrm{C}-\mathrm{H}$ & $\mathrm{r}_{e}=1.09$ \\
$\mathrm{C}-\mathrm{C}$ & $\mathrm{r}_{e}=1.526$ \\
$\mathrm{C}-\mathrm{O}$ (ester) & $\mathrm{r}_{e}=1.41$ \\
$\mathrm{O}($ ester)-S & $\mathrm{r}_{e}=1.58$ \\
$\mathrm{~S}-\mathrm{O}$ & $\mathrm{r}_{e}=1.46$ \\
& \\
O-S-O & $\mathrm{k}_{i j k}=427, \theta_{e}=115.4^{o}$ \\
O-S-O(ester) & $\mathrm{k}_{i j k}=427, \theta_{e}=102.6^{\circ}$ \\
S-O(ester)-C & $\mathrm{k}_{i j k}=418, \theta_{e}=112.6^{o}$ \\
O(ester)-C-H & $\mathrm{k}_{i j k}=418.4, \theta_{e}=109.5^{\circ}$ \\
O(ester)-C-C & $\mathrm{k}_{i j k}=418.4, \theta_{e}=109.5^{\circ}$ \\
H-C-H & $\mathrm{k}_{i j k}=418.4, \theta_{e}=109.5^{\circ}$ \\
H-C-C & $\mathrm{k}_{i j k}=418.4, \theta_{e}=109.5^{\circ}$ \\
C-C-C & $\mathrm{k}_{i j k}=334.7, \theta_{e}=109.5^{\circ}$ \\
& \\
C-C-C-O(ester) & $A_{i j k l}=11.7, \mathrm{~m}=3$ \\
C-C-O-(ester)-S & $A_{i j k l}=9.6, \mathrm{~m}=3$ \\
C-O(ester)-S-O & $A_{i j k l}=2.09, \mathrm{~m}=3$ \\
S-O(ester)-C-H & $A_{i j k l}=1.6, \mathrm{~m}=3$ \\
\hline
\end{tabular}


The simulations involved $5 \times 10^{5}$ steps, i.e., 1 ns. For the 32 surfactant simulations we extracted 200 configurations, which were used later for the single point DFT computations discussed below. All the density/charge profiles presented in this work were averaged out using the data obtained above and below the center of mass of the film.

\subsection{Density Functional Theory computations}

The adiabatic first-principles DFT single point computations were performed using the SIESTA method ${ }^{46}$. SIESTA uses strictly localized atom-centered numerical basis functions to represent the Kohn-Sham orbitals. The interactions between the ionic core and valence electrons are dealt with pseudopotentials. Ionic cores (nuclei and their corresponding core electrons) are described by norm-conserving pseudopotentials in fully factorized form ${ }^{47}$. The electron-electron exchange correlation interactions were computed via DFT using the PBE generalized gradient approximation $(\mathrm{GGA})^{48}$. This represents a good approach to model hydrogen-bonded systems such as water, for the properties explored in this work (see Ref. ${ }^{36}$ ).

Following reference ${ }^{36}$ we employed numerical atomic orbitals to construct the basis set. A real-space grid was used to compute integrals beyond two-body, and the plane wave cutoff for the grid was set to $150 \mathrm{Ry}$. We used double- $\zeta$ polarized atomic orbitals. Hydrogen was represented with two $1 \mathrm{~s}$ and one $2 \mathrm{p}$ orbitals, oxygen and carbon with two $2 \mathrm{~s}$, two shells of $2 p$ and one $3 d$ orbitals, sulfur with two $3 \mathrm{~s}$, two shells of $3 p$ and one $3 \mathrm{~d}$, and finally sodium with two $3 \mathrm{~s}$ and one $3 \mathrm{p}$ orbitals. The double- $\zeta$ polarized bases are confined to spherical finite support of radii of $7 \mathrm{Bohr}$ for $\mathrm{C}, \mathrm{O}$, and $\mathrm{H}$, and $6.5 \mathrm{Bohr}$ for $\mathrm{S}$ and $\mathrm{Na}$. Other defining parameters were obtained variationally as described in ref. ${ }^{49}$. The core of $\mathrm{Na}$ as described by our pseudopotentials includes the $2 s^{2} 2 p^{6}$ shells which are relatively shallow, rendering the pseudopotential less reliable than the others. The structure and dynamics, however, are not affected by it, and the charge deformation discussed below (the relevant magnitude in this study) is sufficiently well described for the purposes of this work.

We performed single point computations of selected configurations generated using classical molecular dynamics. These computations are very computational intensive and we considered the 32 SDS system only. This system already contains 1784 atoms and 4416 valence electrons, which need to be considered explicitly. The computations required about $3 \mathrm{hrs}$ of CPU time per configuration using 32 processors. All the computations were performed using periodic boundary conditions. The length of the box in the plane of the film was the same as that used in the classical simulations, $22.98 \AA$, and the box length in the perpendicular direction was set to $65 \AA$.

The DFT computations were used to compute the density of valence electrons, $\rho_{v}$ in the film. This density was aver- aged out in the plane parallel to the film ${ }^{50}$. The total electron density across the film, $\rho(z)=\rho_{v}(z)+\rho_{c}(z)$, requires the computations of the density of core electrons, $\rho_{c}(z)$. We estimated $\rho_{c}(z)$ from the positions of the atoms obtained in the classical trajectories. The core density on each atom was approximated using a delta function. Subsequently we performed an ensemble average to construct the density profile. We note that for our computations this procedure gives virtually indistinguishable results to those obtained by considering the pseudopotentials.

We have characterized the electrostatic properties of the Newton Black Film through the computation of the total electrostatic potential at a point $\vec{r}, \phi(\vec{r})$. The potential is defined as the sum of the Hartree potential plus the potential describing the electron attraction by the core ions, which is described by the sum of the local parts of the pseudopotentials on the atoms (SIESTA defines the local part of the pseudopotential as the potential generated by the net charge of the core ion spread over a Gaussian of the width of the core). This electrostatic potential is in strict sense, the potential energy felt by one electron in the film, and includes the repulsions associated to other electrons and the attractions arising from the nuclei.

In addition to the total electrostatic potential, we consider a deformation charge potential (DCP) to quantify the electrostatics of the Black Films. The DCP is obtained from the following charge density, $\delta \rho(r)=\rho_{v}(\vec{r})-\rho_{\text {atomic }}(\vec{r})$, where $\rho_{v}(\vec{r})$ is again the valence pseudo-charge density and $\rho_{\text {atomic }}(\vec{r})$ is the sum of the atomic valence pseudo-charge densities. More specifically, $\rho_{\text {atomic }}(\vec{r})$, is the charge density arising from the neutral atoms. This charge density is equivalent to the one that would be obtained from the free atoms, where the electron clouds are not perturbed by other atoms. The DCP directly provides the deviation in charge density of what neutral atoms would give, in analogy with the empirical charges, but also including polarisation and bonding charge-density deformations. The comparison of the DCP potential and the total potential, $\phi(\vec{r})$, provides information on possible ionization and electronic polarization effects at the interfaces and in the film core. Moreover, the DCP provides a measure of the potential prior to any response to the test charge, but on a length scale that does not penetrate into intra-atomic regions. Allowing such penetration (as done with the electrostatic potential obtained directly from DFT), would result in potential 'ramps' associated to intra-atomic dipoles, which are irrelevant to the processes for which this potential is useful.

In addition to the quantum computations of the NBF we have performed ab-initio molecular dynamics computations of a thin slab of water. The water surface has been investigated before ${ }^{37,38}$ using DFT computations. We will use these results to assess the reliability of our computations of the electrostatic potentials. The simulations involved 64 water molecules in a prismatic box of dimensions, $L_{z} / 2=L_{x}=L_{y}=12.63 \AA$ with 
full periodic boundary conditions. The water slab was simulated at constant temperature using the Nosé thermostat with a time step of $0.5 \mathrm{fs}$. We performed the simulations at a high temperature, $360 \mathrm{~K}$, as suggested in Ref. ${ }^{36}$ and as it has become common practice in first-principles simulations of wet systems based on GGA, to recover good descriptions of the structural and diffusivity properties of liquid water (there is a known GGA anomaly for liquid water description, which slightly overestimates the energy scale of the effective barriers for liquid diffusion) ${ }^{36}$. The simulations were performed with the code SIESTA. The treatments of the pseudopotentials, exchange correlation interactions and numerical atomic orbitals was done at the same level of theory discussed above for the single point computations. We generated a trajectory of 15 ps, and ten configurations were selected for the computation of the average electrostatic potential, $\phi(\vec{r})$, which is defined as before.

\section{Results}

Figure 2 show the density profiles of the Newton Black Film obtained from the analysis of the classical trajectories. We compare results obtained using 32 and 128 surfactants, which correspond to a box length on the film plane of $L=22.98$ $\AA$ and $L=45.96 \AA$ respectively. In this way we can test the accuracy of the small system in describing the Newton Black Film structure. The small system reproduces well the general structure of the NBF (see Figure 2). The more noticeable difference is that the density profiles are more localized and the carbon atoms density profile shows more structure. These differences are connected to the blurring effect of the capillary waves, which according to capillary wave theory increases logarithmically with the box length ${ }^{51}$. This dependence with the box length breaks down for small simulation boxes, typically $L<8-10 \AA$ in ionic interfaces ${ }^{39}$, because the long wave length thermal fluctuations are inhibited by the simulation box size. Chacón and Tarazona have recently shown that it is possible to compute the so called intrinsic density profile, which eliminates the thermal averaging effect of the capillary waves, providing a direct view of the real structure of the interface. The resulting profile is more structured resembling the pair correlation function of a dense fluid ${ }^{52-55}$. For the specific case of alkane interfaces (dodecane) it was found that the effective molecular cut-off area for the capillary wave spectrum is of the order of $A \approx 350 \AA^{2}$ at $300 \mathrm{~K}$. The area of the interface of the small system considered here, $A=529 \AA^{2}$, is of the same order, and much smaller than the area, $A=2466 \AA^{2}$, of the 128 surfactant system. This supports the conclusion that the effect of the thermal capillary waves is reduced in our small system containing 32 surfactants.

In agreement with previous work on liquid alkanes ${ }^{43,44}$, we find that the structure of the film modeled either with par-
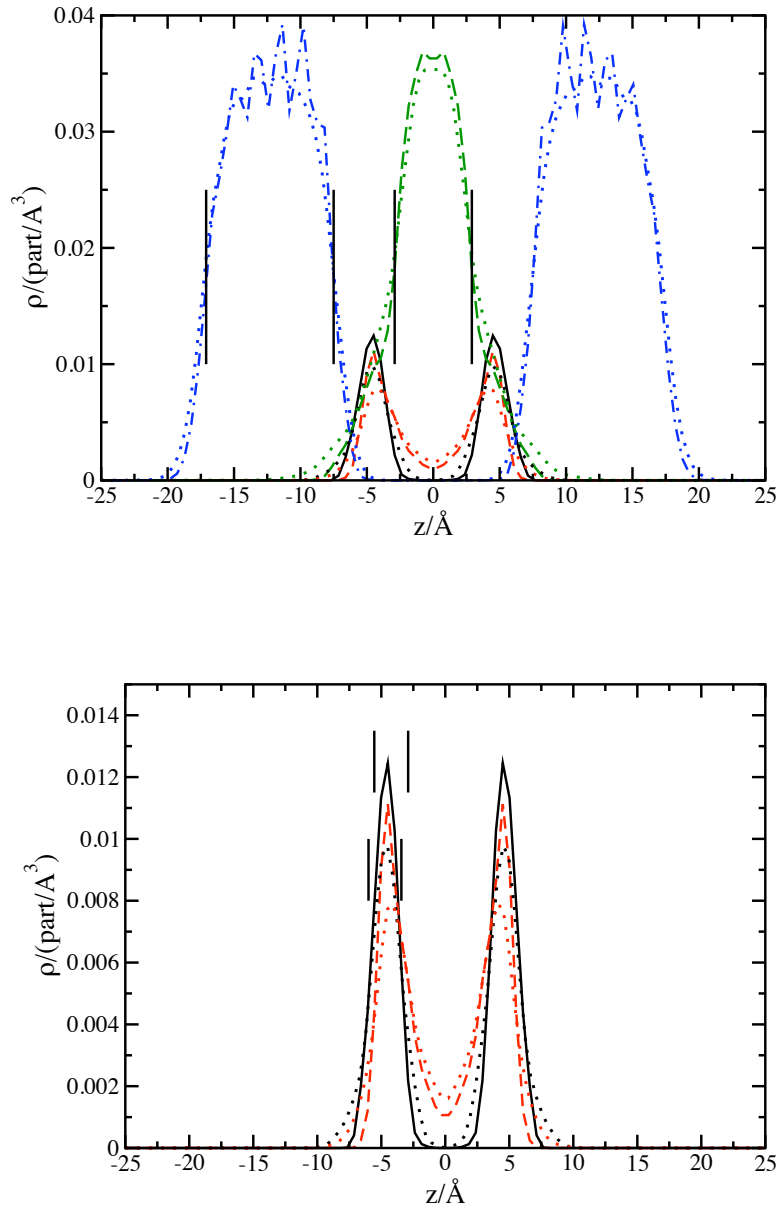

Fig. 2 Density profiles of the Newton Black Film investigated in this work. The results correspond to the NQ-SDS empirical force-field model. We compare the results of the 32 SDS (full line: sulfur; short dashed line: sodium; long dashed line: water oxygen; dot-dashed line: carbon atoms) and the 128 SDS system (dotted lines). (Top) water molecules, all carbon atoms, sulfur and sodium and (bottom) sulfur and sodium only. The vertical lines represent the approximate thickness of the different regions in the film; aliphatic chain, sulfur, sodium and water core. The location of the lines corresponds to half the maximum density of the corresponding density profile.

tial charges on the carbon and hydrogen atoms (Q-SDS - not shown) or without partial charges (NQ-SDS), is essentially the same.

The film thickness (taken as the distance between the hydrocarbon-vacuum interfaces - see vertical lines in figure 2 ) is $\approx 34 \AA$, which is similar to the values estimated using Xray reflectivity experiments ${ }^{8}, 32.9 \pm 0.5 \AA$. In agreement with 
previous work ${ }^{10,22,23}$, we find that the $\mathrm{Na}^{+}$counterions are strongly adsorbed at the head groups. It was found that about 2.3 water molecules are solvating each head group ${ }^{22}$. The remaining water in our film is distributed in the film core in a well defined layer whose density corresponds to the bulk water density $\approx 0.033$ molecules $/ \AA^{3}$. There are three well defined regions in this film, namely, the hydrocarbon chain, the aqueous core and the head group and counterions layer (see vertical lines in Figure 2). We will refer to these three regions later on, when discussing the electronic structure of the film.

Figure 3 shows the electrostatic potential obtained from the classical simulations. This potential arises from the counterions, water and SDS partial charges. The electrostatic field, $\mathrm{E}(\mathrm{z})$, and the 'classical' electrostatic potential, $\phi(z)$, were obtained using Gauss's theorem,

$$
\frac{-d \phi_{\text {classical }}}{d z}=E(z)=\frac{1}{\varepsilon_{0}} \int_{-\infty}^{z} \rho\left(z^{\prime}\right) d z^{\prime}
$$

where $\rho\left(z^{\prime}\right)$ is the electrostatic charge density at the position $z^{\prime}$. We have represented in Figure 3 two contributions to the charge density, one from the free charges, i.e., counterions and surfactants, and that of the partial charges in the SPC/E water molecules. Our data supports previous observations regarding the existence of an anomalous dielectric behavior in interfacial water ${ }^{10,23}$, namely, the water molecules contribute with a field that has opposite sign to that originated from the ions and the head groups (free charges). As expected, the electrostatic fields arising from the head groups-counterions and the solvent are fairly insensitive to the force-field used to model the surfactants, either Q-SDS or NQ-SDS (see Figure 3), but we find that the inclusion of partial charges to model the aliphatic chain adds a non-negligible contribution to the electrostatic potential in the SDS-vacuum interface region. We find a negative potential, $\approx-0.4 \mathrm{~V}$, which is of the same order as that obtained for the water surface using empirical force-fields. The negative sign indicates an excess of positive charge at the interface. This potential arises from the charge separation in the $\mathrm{CH}_{3}$ terminal groups, and spans $\approx 4 \AA$, indicating certain degree of disorder in the aliphatic-vacuum interface.

We emphasize that the 'classical' electrostatic potential represented in Figure 3 arises from the distribution of charges in the film alone, and unlike the 'ab initio' potential, does not include electronic polarization and electronic delocalization effects. This difference has to be taken into account when comparing classical and 'ab initio' electrostatic potentials.

Figure 4 shows our first result comparing classical and ' $a b$ initio' data. We discuss in the following the electron density distribution in the NBF. The classical density profile can be obtained from the atomic density profiles and the total number of electrons in each atom. The total number of electrons was corrected using the partial charges defined in the NQ-SDS force-field. The DFT theory provides information on the den-

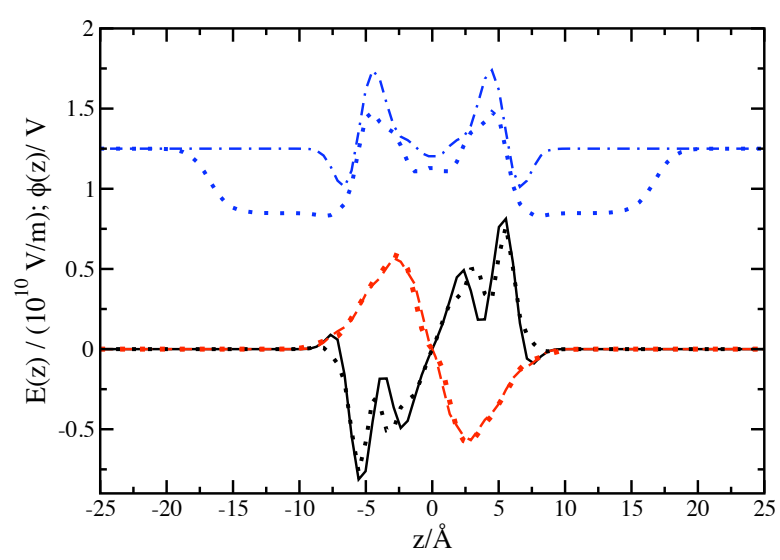

Fig. 3 Electrostatic field contributions and total electrostatic potential for the NQ-SDS (no charges in the aliphatic chain) and Q-SDS (charges) force-fields. Lines represent the NQ-SDS results: water (dashed line), free charges -head group and sodium counter ions- (full line). The dots represent the corresponding Q-SDS data. The total electrostatic potentials (dot-dashed line and dots) have been shifted vertically by 1.25 units for greater clarity.

sity of the valence electrons only. In order to compute the total electronic density we have computed the density of core electrons using the atomic positions obtained in the classical trajectory (see Methodology above). The core contribution is more important in the surfactant head group, whereas in the hydrocarbon chains it adds about $1 / 4$ of the total electronic density (see Figure 4). The DFT and classical density profiles are almost identical in the scale of the figure.

In Figure 4, we compare our electron density profiles with a schematic view of the experimental X-ray data. The experimental result is based on a five-lamina model, consisting of two aliphatic, two polar head and one aqueous core region 8,56 . Our density profiles agree well with the X-ray prediction, particularly in the aliphatic region, whose intensity is determined by the alkanes tilt angle. The simulations predict more localization in the position of the polar heads resulting in a slightly higher intensity in the density profile.

Figure 5 shows the total electrostatic potential obtained from Density Functional Theory. We recall that this potential includes the Hartree potential and the local pseudopotential. The electrostatic charge separation between the head group and the counterions (see $\mathrm{S}$ and $\mathrm{Na}$ in Figure 5) results in very strong oscillations in the electrostatic potential. The general shape of the DFT potential is similar to the classical electrostatic potential (c.f. Figure 3), although the potential differences between different regions in the film are much larger, 


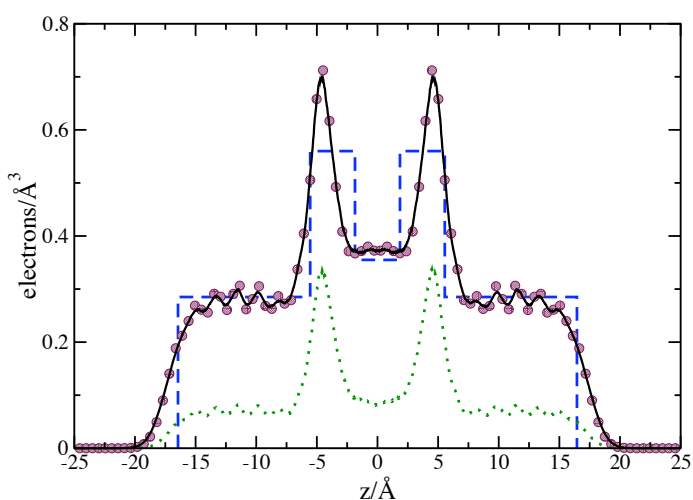

Fig. 4 Electron density profile of sodium dodecyl sulfate. Circles: classical simulations using the NQ-SDS force-field; full line: DFT computations obtained from the configurations generated using classical molecular dynamics simulations; dotted line: core electron contribution obtained from the classical molecular dynamics trajectory; dashed lines: experimental estimate of the electron density profile inferred from X-ray reflectivity measurements (see text and ref. ${ }^{8}$ ).

typically one order of magnitude larger than the classical one. Such differences between classical and DFT potentials have been discussed before in electronic structure computations of the water surface ${ }^{37,38}$. DFT predicts a significant drop in the electrostatic potential, $\approx 2 \mathrm{~V}$, in going from the head group region to the aqueous core and about $\approx 4 \mathrm{~V}$, in the SDS-vacuum interface. The latter value is in line with recent computations for water ${ }^{37,38}$. Considering that slow ions as test charges cannot probe the inner regions of atoms, we doubt this potential drop is relevant to typical surface-potential experiments, although it is important for electron probes.

Towards an analysis more comparable with what can be obtained with classical atom charges and what can be measured with slow ion test charge experiments, we have further computed the deformation charge potential (DCP). As discussed above this potential arises from the difference between the total charge density and the charge density of the free unperturbed atoms. The DCP potential should be constant whenever there is no charge displacement due to electronic polarization, ionization or bonding. We find that this potential features a drop of $\approx-1 \mathrm{~V}$ at the SDS-vacuum interface (c.f. Figure 5). This indicates a defect of electrons (or a positive charge) at the SDS-vacuum interface, which is in line with the results obtained from the classical computations, using the empirical force-field that incorporates partial charges in the aliphatic region (Q-SDS). We emphasize though, that the electrostatic potential obtained in the classical simulations arises from the

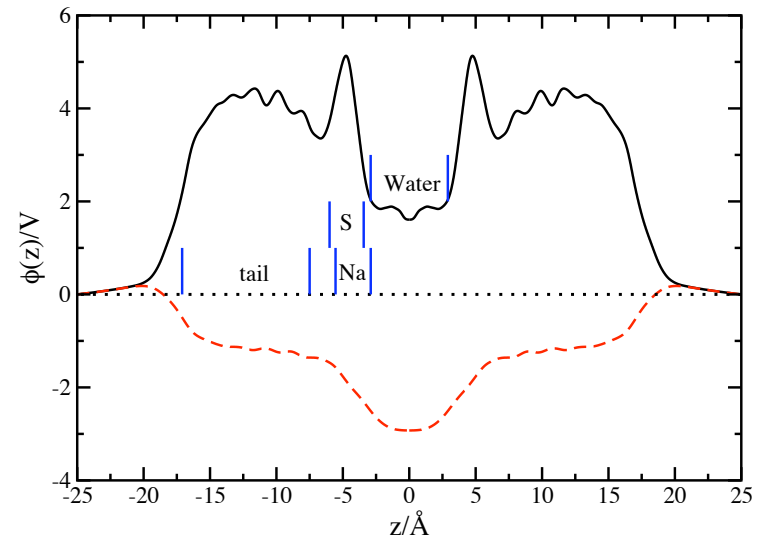

Fig. 5 Total electrostatic potential obtained from DFT computations (full line), and the deformation charge potential obtained by subtracting the valence pseudocharge density and the sum of atomic valence pseudocharge densities (dashed line). The vertical lines indicate the location of the different atoms or groups in the SDS molecule. See figure 2 .

break in symmetry associated to the SDS-vacuum interface and the partial charges in the carbon and hydrogen atoms, hence it does not include polarization effects such as charge transfer. Interestingly, we find that the DFT provides evidence for the existence of charge transfer along the SDS chain. The DCP potential is not constant in the aliphatic region and features a small drop of $\approx-0.4 \mathrm{~V}$ in going from the terminal group to the head group. The possibility of charge transfer effects in these surfactants has been discussed before. Using first-principles computations it has been shown that anionic hydrocarbons such as SDS feature significant charge transfer, which extends from the head group to the terminal methyl group 57,58 . Semiempirical methods indicate that such charge transfer also occurs in normal alkanes, although in the case of dodecane, which is relevant to our system, the charge transfer results in charge separation in the two terminal methyl and methylene groups.

The DCP shows a larger drop $\approx-1.53 \mathrm{~V}$ at the head-group aqueous core interface. We find that the potential is essentially flat in the center of the core, which indicates a lack of significant electronic polarization in the aqueous region. This result is relevant to validate recent work using classical potentials, where it has been reported that water exhibits an anomalous dielectric behavior when it is confined in Newton Black Films ${ }^{10}$. In that work the 'polarization' effects can arise from the solvent reorientation 'only', as electronic degrees of freedom are not included. The lack of significant electronic polar- 
ization effects supports the view that water reorientation may contribute significantly to the electrostatics of water confined in the NBF.

To gain further insight on the DFT electrostatic potentials we have revisited the problem of the water surface. This has been investigated recently using two different approaches; 1) Car-Parrinello Molecular Dynamics at $\mathrm{T}=298 \mathrm{~K},{ }^{37}$ using the BLYP functional, and 2) a mixed classical-DFT analysis similar to the one employed here, whereby the trajectory is generated using a classical force-field at $\mathrm{T}=300 \mathrm{~K}$, and the electrostatic potential is computed with DFT using the PBE functional ${ }^{38}$. Despite the rather different approach these two works predict electrostatic potentials of the same order, +3.1 $\mathrm{V}^{37}$ and $+3.63 \mathrm{eV}^{38}$. Our results were obtained using ab initio Molecular Dynamics following the method discussed in section 2.2. Our electrostatic potential confirms previous computations, it is positive and of the order of $3 \mathrm{eV}$. Within the statistical uncertainty of our computations, the DCP potential is approximately flat in the centre of the film, and it features a significant drop at the water-vacuum surface. The magnitude of the drop associated to the water surface is $-1 \mathrm{eV}$. This value agrees in sign and it is closer in magnitude to the electrostatic potential obtained from classical simulation using empirical force-fields.

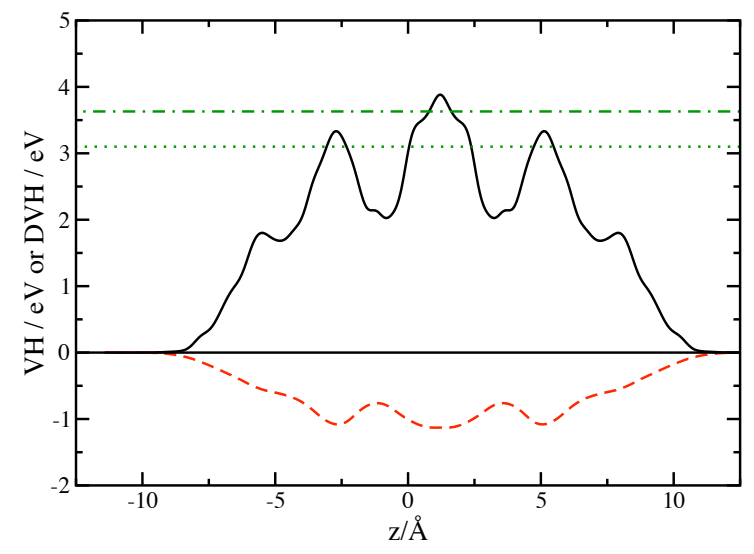

Fig. 6 The total electrostatic potential (full line) and the deformation charge potential (dashed line) of the water surface obtained from DFT ab initio simulations. The horizontal line represents recent estimates of the surface potential obtained in references ${ }^{37}$ (dots) and ${ }^{38}$ (dash-dotted line) using ab-initio Car-Parrinello molecular dynamics and a mixed DFT-classical simulation approach respectively. See main text for details.

\section{Conclusions and Final Remarks}

We have performed classical and ab initio computations of Newton Black Films (NBF) made of sodium dodecyl sulfate surfactants and water. To our knowledge this is the first quantum mechanical investigation of this important class of soft interfaces, which feature in gas-liquid and liquid-liquid dispersions. The ab-initio simulation of these systems is very time consuming. To ensure adequate sampling and capture the thermal fluctuations of the film we generated classical trajectories using empirical force-fields. The electronic properties of the film were later investigated using Density Functional Theory.

The film structure obtained from classical simulations is fairly insensitive to the details of the force-field, in particular to whether partial charges are included or not in the modeling of the surfactant aliphatic chains. However, the presence of partial charges adds a non-negligible contribution to the electrostatic potential at the surfactant-vacuum interface, $-0.4 \mathrm{~V}$, which is of the same order as the electrostatic potential of the water surface.

The electron density profiles of the NBF obtained from DFT are consistent with the experimental interpretation of the $\mathrm{X}$ ray reflectivity experiments. Overall, we find that the combination of classical trajectories and DFT electron structure computations provides an accurate approach to predict the electron distribution of soft interfaces.

The DFT electrostatic potential of the NBF features large drops at the interfaces, $\approx 2-4 \mathrm{eV}$. These values are similar to the ones observed in free water surfaces, $3-3.5 \mathrm{~V}$. The fact that the quantum fluctuations of electrons into vacuum are still larger than the corresponding thermal fluctuations of the nuclei may contribute to the magnitude of these electrostatic potentials. Considering that slow ions as test charges cannot probe the inner regions of atoms, we doubt this potential drop is relevant to typical surface-potential experiments, although it is important for electron probes.

In order to advance in the investigation of interfacial electrostatic potentials, we have considered the deformation charge potential DCP. The DCP predicts charge separation at the SDS-vacuum interface. This results is consistent with that obtained using classical force-fields that incorporate partial charges to model the SDS aliphatic chain. Moreover, the DCP predicts that the aliphatic region of the NBF is weakly polarized. This result agrees with semiempirical computations of single anionic surfactants, and supports the view that the interior of self assembled structures made of SDS surfactants, e.g. micelles, may be polar ${ }^{58}$. An interesting consequence of these polarization effects would be and increase in the penetration of water and ions in the hydrophobic region of the NBF.

Finally, we have shown that the deformation charge potential of a water slab simulated using ab initio molecular dynamics simulations, is negative and of the order of $-1 \mathrm{~V}$. This po- 
tential is in closer agreement with the electrostatic potentials obtained from classical force-field simulations We believe that the deformation charge potential provides a suitable approach to compare the electrostatic properties of quantum and classical simulations.

\section{Acknowledgements}

We thank Michiel Sprik and José M. Soler for valuable discussions on the interpretation of the ab initio electrostatic potentials, and the Imperial College High Performance Computing Service for providing computational resources.

\section{References}

1 D. F. Evans and H. Wennerström, The Colloidal Domain, Wiley-VCH, 2nd edn, 1999.

2 V. J. Bergeron, J. Phys. Condens. Matter, 1999, 11, R215-R238.

3 M. N. Jones, K. J. Mysels and P. C. Sholten, Trans. Faraday Soc., 1966, 62, 1336-1348

4 V. J. Bergeron, A. Waltermo and P. M. Claesson, Langmuir, 1996, 12, 1336-1342.

5 A. Waltermo, P. M. Claesson, S. Simonsson, E. Manev, I. Johansson and V. J. Bergeron, Langmuir, 1996, 12, 5271-5278.

6 J. Israelachvili, Intermolecular and Surface Forces, Academic Press, 2nd edn, 1991.

7 D. Exerowa, T. Kolarov and K. H. Khristov, Colloids and Surfaces, 1987, 22, 171-185

8 O. Bélorgey and J. J. Benattar, Phys. Rev. Lett., 1991, 66, 313-316.

9 B. W. Ninham, Adv, Colloid Interface Sci., 1999, 83, 1-17.

10 J. Faraudo and F. Bresme, Phys. Rev. Lett., 2004, 23, 236102.

11 S. Leikin, V. A. Parsegian, D. C. Rau and R. P. Rand, Annu. Rev. Phys. Chem., 1993, 44, 369-395.

12 S. Marcelja and N. Radic, Chem. Phys. Lett., 1976, 42, 129-130.

13 N. A. Besseling, Langmuir, 1997, 13, 2113-2122.

14 J. N. Israelachvili and H. Wennerström, Langmuir, 1990, 6, 873-876.

15 J. N. Israelachvili and H. Wennerström, J. Phys. Chem., 1992, 96, 520 531.

16 J. N. Israelachvili and H. Wennerström, Nature (London), 1996, 379, 219-225.

17 E. Ruckenstein and M. Manciu, Langmuir, 2002, 18, 7584-7593.

18 J. Faraudo and F. Bresme, Phys. Rev. Lett., 2005, 94, 077802.

19 C. Hédreul and G. Frens, Colloids and Surfaces A: Physicochemical and Engineering Aspects, 2001, 186, 73-82.

20 P. Poulin, F. Nallet, B. Cabane and J. Bibette, Phys. Rev. Lett., 1996, 77, 3248-3251.

21 Z. Gamba, J. Hautman, J. C. Shelley and M. L. Klein, Langmuir, 1992, 8, 3155-3160.

22 F. Bresme and J. Faraudo, Langmuir, 2004, 20, 5127-5137.

23 F. Bresme and J. Faraudo, Molecular Simulation, 2006, 32, 1103-1112.

24 W. H. Yang, R. L. Wu, B. Kong, X. F. Zhang and X. Z. Yang, J. Phys. Chem. B, 2009, 113, 8332-8338.

25 S. D. Napoli and Z. Gamba, J. Chem. Phys., 2010, 132, 075101.

26 P. Fenter and N. C. Sturchio, Prog. Surf. Sci., 2004, 77, 171.

27 P. Junwirth and D. J. Tobias, Chem. Rev., 2006, 106, 1259-1281.

28 N. T. Skipper, P. A. Lock, J. O. Titiloye, J. Swenson, Z. A. Mirza, W. S. Howells and F. Fernández-Alonso, Chemical Geology, 2006, 230, 182 196.
29 M. Sprik, J. Hutter and M. Parrinello, J. Chem. Phys., 1996, 105, 1142 1152.

30 J. Ortega, J. P. Lewis and O. Sankey, J. Chem. Phys., 1997, 106, $3696-$ 3702.

31 P. L. Silvestrelli and M. Parrinello, Phys. Rev. Lett., 1999, 82, 3308-3311.

32 S. Izvekov and G. A. Voth, J. Chem. Phys., 2002, 116, 10372-10376.

33 J. C. Grossman, E. Schwegler and E. W. Draeger, J. Chem. Phys., 2004, 120, 300-311.

34 E. Schwegler, J. C. Grossman, F. Gygi and G. Galli, J. Chem. Phys., 2004, 121, 5400-5409.

35 D. Asthagiri, L. R. Pratt and J. D. Kress, Phys. Rev. E, 2003, 68, 041505.

36 M. V. Fernández-Serra and E. Artacho, J. Chem. Phys., 2004, 121, 1113611144.

37 S. M. Kathmann, I. F. W. Kuo and C. J. Mundy, J. Am. Chem. Soc., 2009 131, 17522-17522.

38 K. Leung, Phys. Chem. Lett., 2010, 1, 496-499.

39 M. González-Melchor, F. Bresme and J. Alejandre, J. Chem. Phys., 2005, 122, 104710

40 H. J. C. Berendsen, J. R. Grigera and T. P. Straatsma, J. Phys. Chem., 1987, 91, 6269-6271.

41 J. Shelley, K. Watanabe and M. L. Klein, Int. J. Quantum Chem: Quantum Biol. Symp., 1990, 17, 103-117.

42 W. L. Jorgensen, D. S. Maxwell and J. Tirado-Rives, J. Am. Chem. Soc., 1996, 118, 11225-11236.

43 B. Chen, M. G. Martin and J. I. Siepmann, J. Phys. Chem. B, 1998, 102, 2578-2586.

44 F. Bresme, E. Chacón and P. Tarazona, Molecular Physics, 2010, 108, 1887-1898

45 W. D. Cornell and et.al., J. Am. Chem. Soc., 1995, 117, 5179-5197.

46 J. M. Soler, E. Artacho, J. D. Gale, A. García, J. Junquera, P. Ordejón and D. Sánchez-Portal, J. Phys.: Condens. Matter, 2002, 14, 2745-2779.

47 N. Troullier and J. L. Martins, Phys. Rev. B, 1991, 43, 1993-2006.

48 J.P. Perdew, K. Burke and M. Emzerhof, Phys. Rev. Lett., 1996, 77, 38653868 .

49 E. Artacho and et.al., J. Phys.: Condens. Matter, 2008, 20, 064208.

50 J. Junquera and P. Ordejón, MACROAVE 1.2.1, 2003

51 R. Evans, Adv. Phys., 1979, 28, 143.

52 E. Chacón and P. Tarazona, Phys. Rev. Lett., 2003, 91, 166103.

53 P. Tarazona and E. Chacón, Phys. Rev. B, 2004, 70, 235407.

54 F. Bresme, E. Chacón, P. Tarazona and K. Tay, Phys. Rev. Lett., 2008, 101, 056102 .

55 F. Bresme, E. Chacón and P. Tarazona, Phys. Chem. Chem. Phys., 2008, 10, 4704-4715.

56 J. J. Benattar and A. Schalchli, Physica Scripta, 1994, 50, 188-194.

57 S. R. Gadre and S. S. Pingale, Chem. Comm., 1996, 5, 595-596.

58 P. D. T. Huibers, Langmuir, 1999, 15, 7546-7550. 


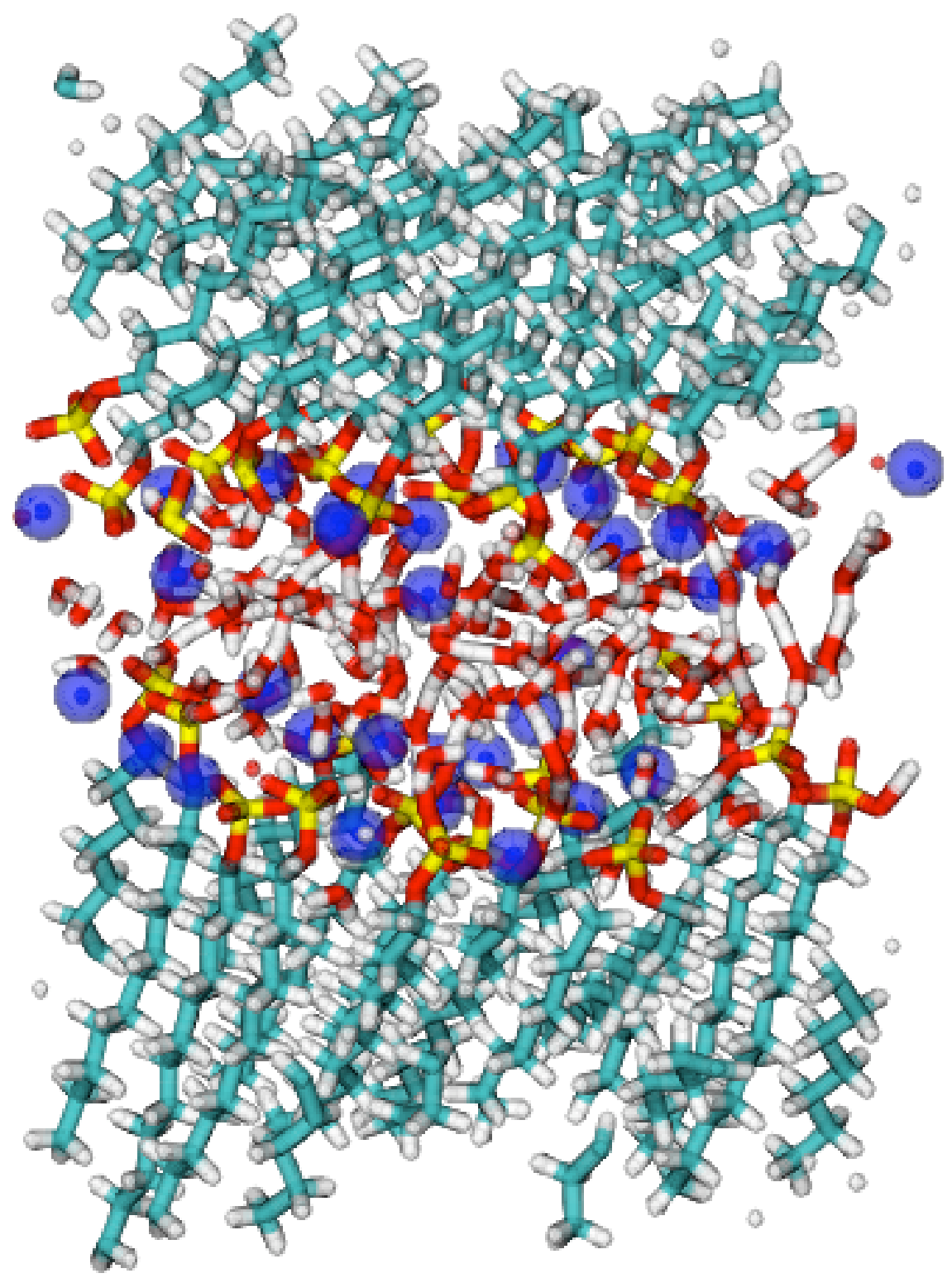




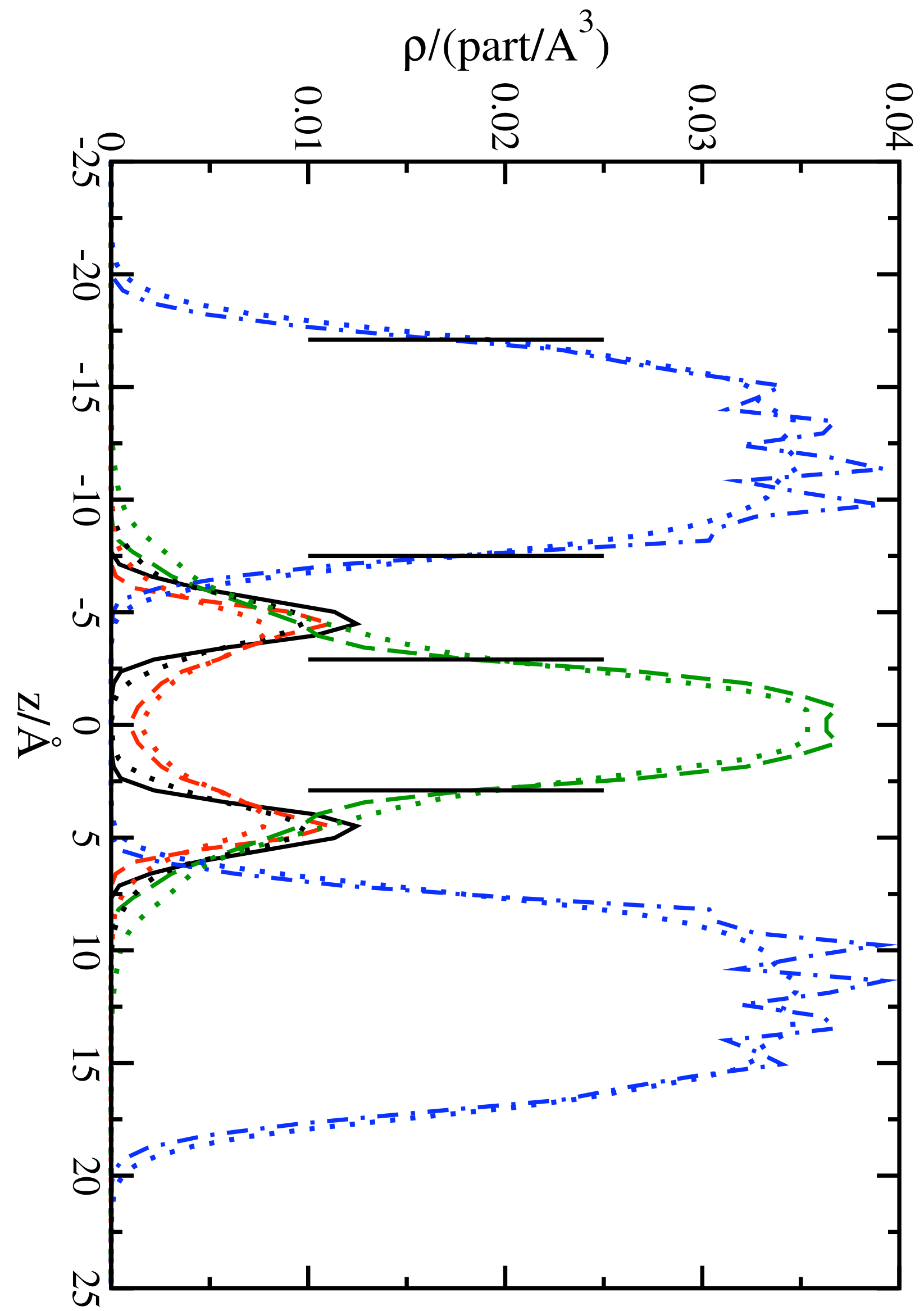

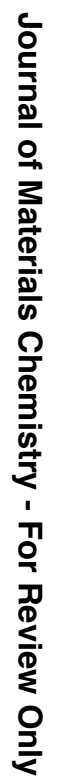




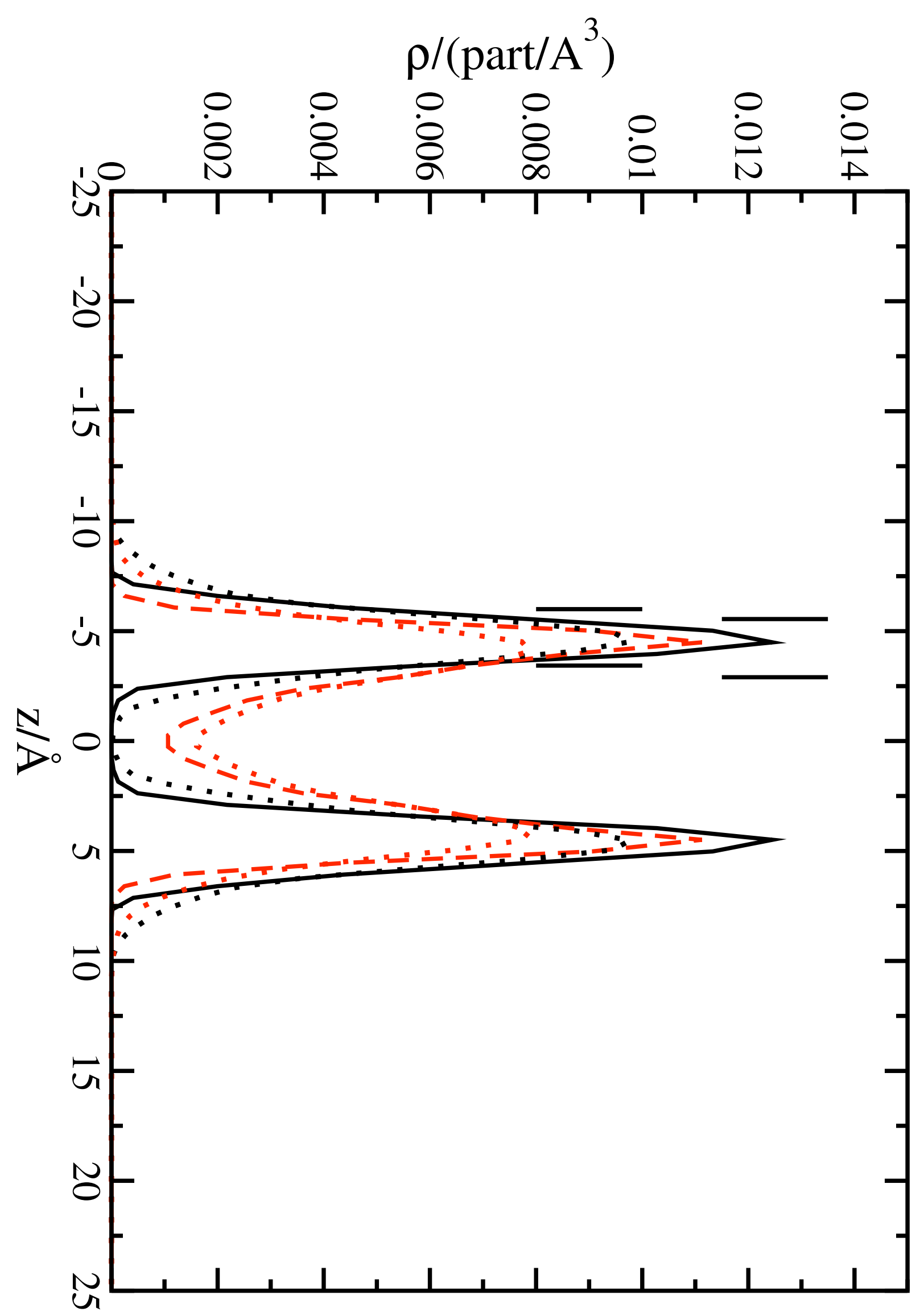




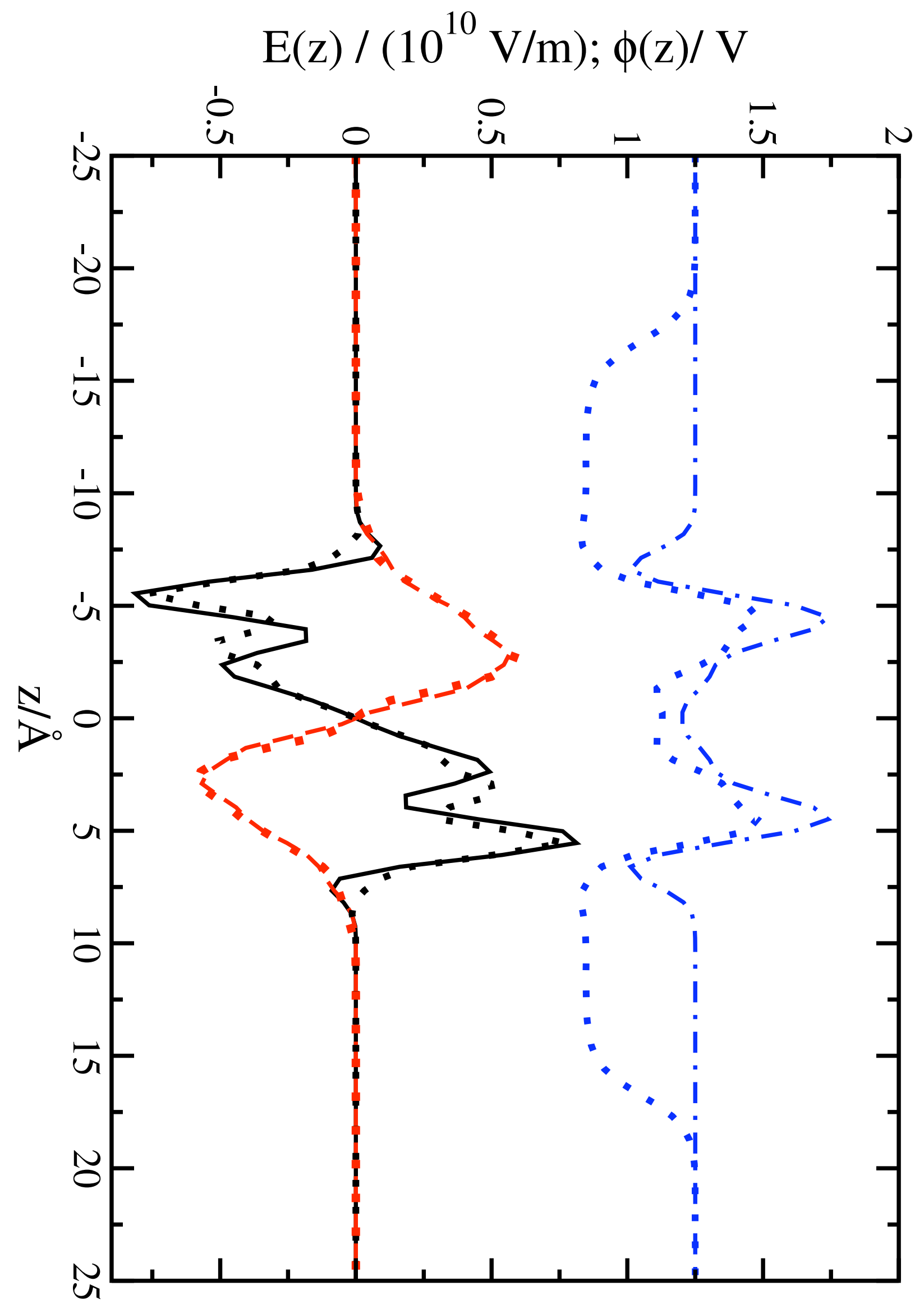

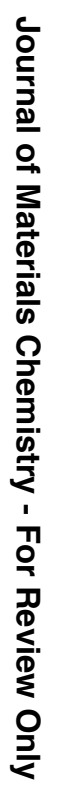




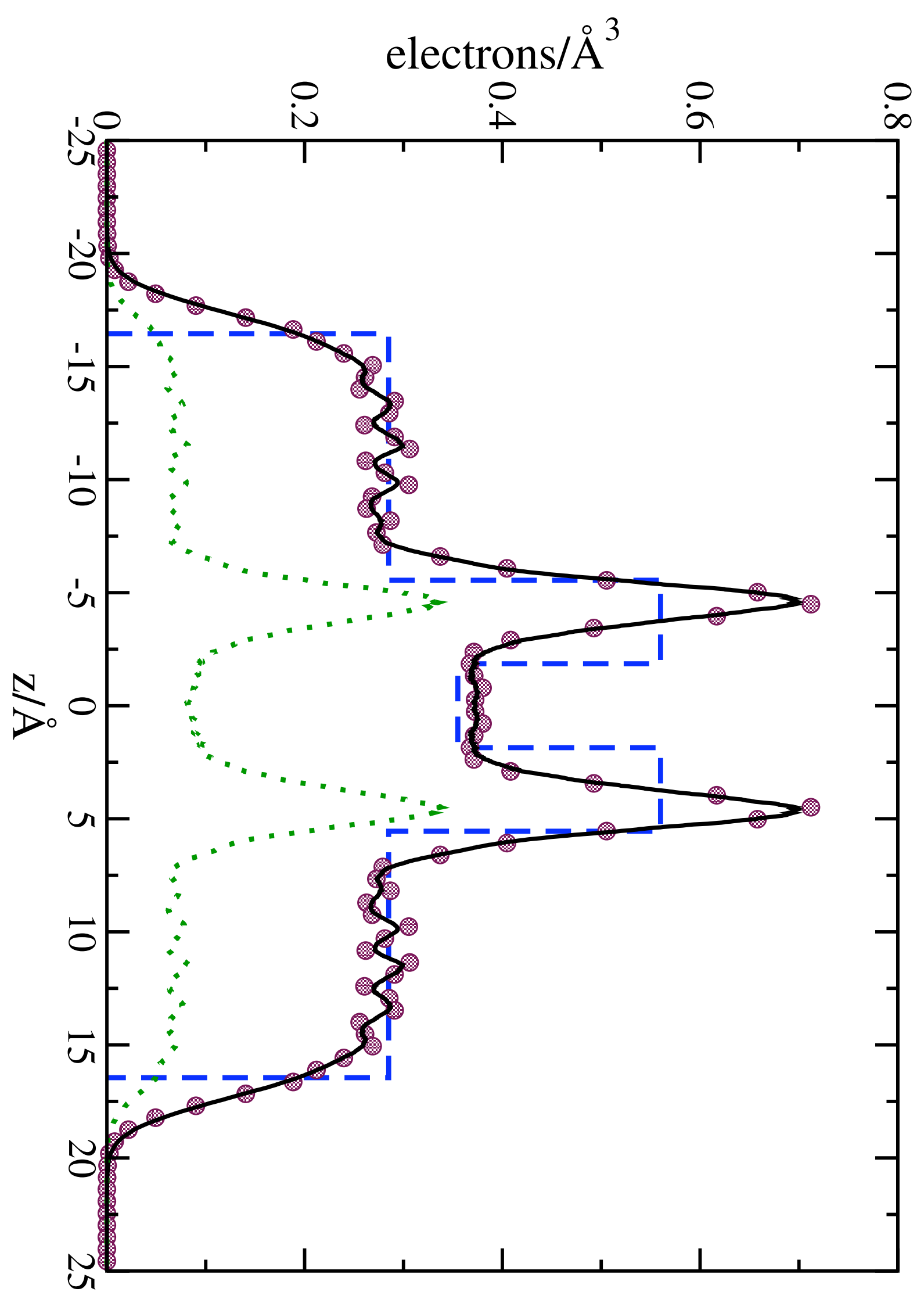

0
0
0
0
0
0
0
$\infty$

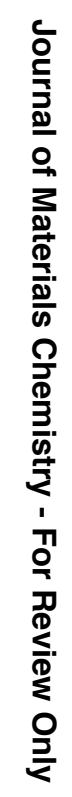




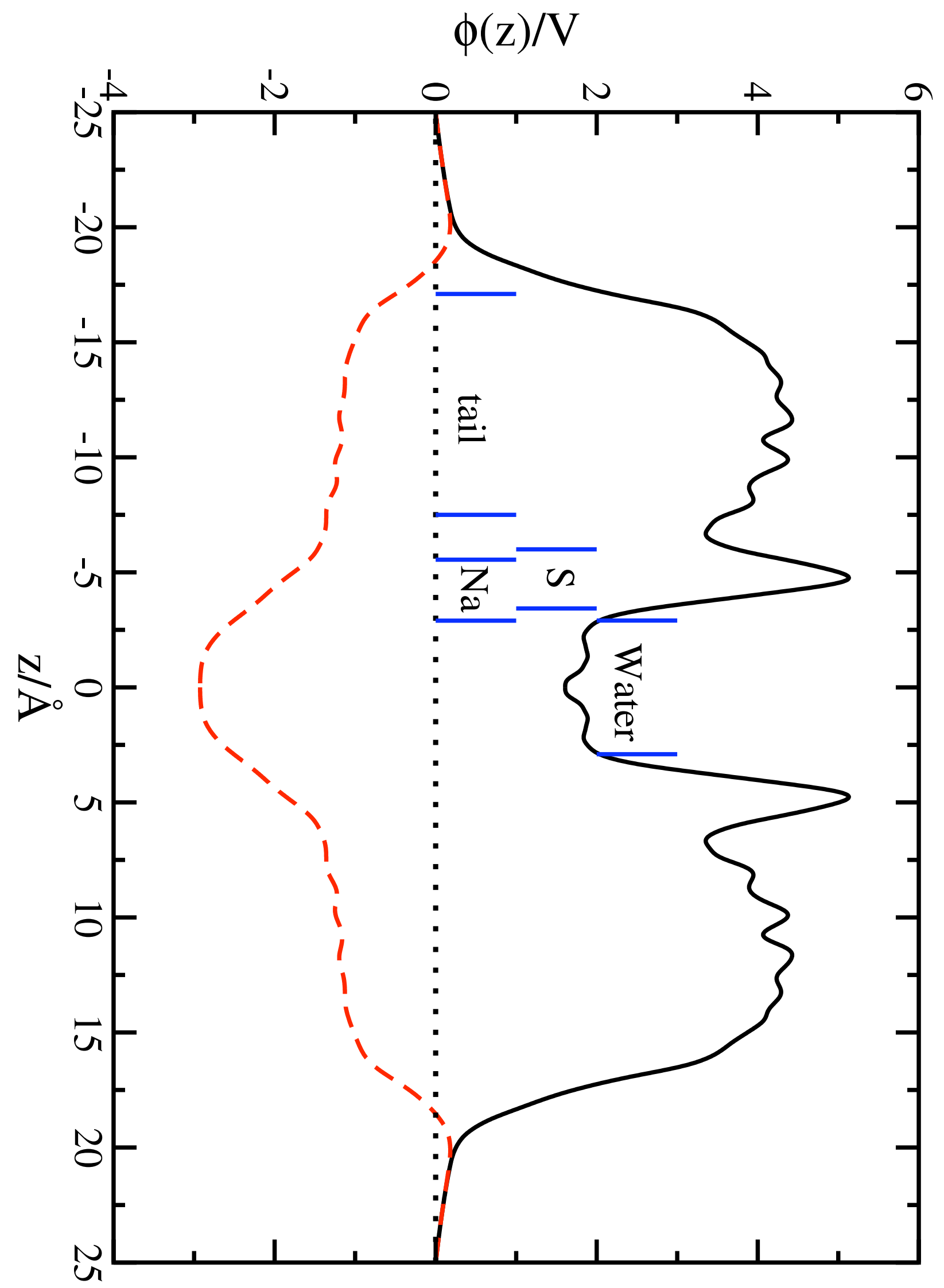

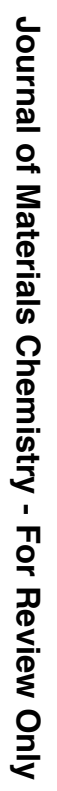

